\title{
Generalized Rank Mapped Transmuted Distribution for Generating Families of Continuous Distributions
}

\author{
M. A. Ali ${ }^{1}$, Haseeb Athar ${ }^{2, *}$ \\ ${ }^{1}$ Department of Statistics, Faculty of Science, King Abdulaziz University, Jeddah, Kingdom of Saudi Arabia \\ ${ }^{2}$ Department of Statistics and Operations Research, Aligarh Muslim University, Aligarh, India
}

\section{ARTICLE INFO}

\section{Article History}

Received 07 Oct 2018

Accepted 05 Jan 2021

Keywords

Transmuted map

Order Statistics

Weibull distribution

Beta distribution

Hazard function

Continuous distributions

AMS Subject Classification:

Primary: 62E15, Secondary: 62F30.

\begin{abstract}
This study introduces generalized transmuted family of distributions. We investigate the special cases of our generalized transmuted distribution to match with some other generalization available in literature. The transmuted distributions are applied to Weibull distribution to find generalized rank map transmuted Weibull distribution. The distributional characteristics such as probability curve, mean, variance, skewness, kurtosis, distribution of largest order statistics, and their characteristics studied to compare with ordinary Weibull distribution. Hazard rate functions and distributional characteristics of largest order statistics of transmuted distributions are also studied. It is observed that the transmuted distributions are more flexible to model real data, since the data can present a high degree of skewness and kurtosis. If someone is interested to locate more flexible and higher degree of skewed distribution can explore this generalized transmuted family of distributions for future use.
\end{abstract}

(C) 2021 The Authors. Published by Atlantis Press B.V. This is an open access article distributed under the CC BY-NC 4.0 license (http://creativecommons.org/licenses/by-nc/4.0/).

\section{INTRODUCTION}

The ideas of developing new distributions are important issues in recent literatures. Numerous families of distributions have proposed by several authors for modeling data in several areas such as engineering, economics, finance and actuarial science, medical and life sciences. However, in many applied areas like lifetime analysis, insurance analysis we need extended distributions, that is, new distributions which are more flexible to model real data, since the data can present a high degree of skewness and kurtosis.

Lee et al. [1] provided an overview of most method used to generate family of continuous distributions earlier in 1980. For more details about these methods can be referred to Pearson [2], Johnson [3], and Tukey [4]. Recently several literatures were discussed generalized method to generate extended generalized family of distributions. For more details about the recent development may refer to Johnson et al. [5], Eugene et al. [6], Jones [7], Alzaatreh et al. [8], Bourguignon et al. [9], Afify et al. [10], Granzotto et al. [11], Al-Kadim and Mohammed [12], Jayakumar and Babu [13], Mahdavi and Kundu [14], Alizadeh et al. [15], Al-Kadim [16], Pobocikova et al. [17], Elgarhy et al. [18], Afify et al. [19], and references therein. Apart from the above, a more extended generalized $n t h$ degree transmuted method suggested in this study to generate transmuted distributions. An application of the generated transmuted map is extended to the Weibull distribution. The distributional characteristics of the generated transmuted distributions are also simulated to compare with traditional Weibull distribution.

This paper is organized in the following way: In Section 2, we developed $n$th degree generalized transmutation map based on continuous family of distribution. Particular cases are also discussed. Some other members of generalized transmutation map are identified. In Section 3 , the survival function, hazard rate function, and reserved hazard rate function of newly generated generalized transmuted distribution are discussed. In Section 4, we developed the $n$th degree generalized transmutation map based on Weibull distribution. Also, probability density function ( $p d f$ ) graphs are simulated and presented in figure to compare each other. In Section 5, some distributional characteristics such as mean variance, skewness, and kurtosis are simulated and presented in tabular form to compare each other for different parametric set of values. The Section 6 is based on discussion and proof of some theorems related to order statistics (OS) of generalized transmuted Weibull distribution (TWD). Simulated distributional characteristics of largest OS of quadratic TWD are also presented in section 6. The Section 7, states conclusion and then inserted references. 


\section{GENERALIZED $n$th RANK TRANSMUTATION MAP}

The construction of the generalized $n$-degree transmutation map considered here is simple and intuitive. Let $X_{1}, X_{2}, \ldots, X_{n}$ be a random sample from an absolutely continuous population with $p d f g(x), x \in(a, b)$ corresponding to cumulative distribution function $(c d f) G(x)$ with $\int_{a}^{b} f(x) d x=\int_{\varphi}^{\psi} f[h(t)] h^{\prime}(t) d t ; x=h(t)$. This is valid under the following conditions:

i) $f(x)$ is continuous on some interval $A \leq x \leq B$ containing the original limits of integration, $(a, b)$.

ii) The equalities $a=h(\varphi)$ and $b=h(\psi)$ holds.

iii) $\quad h(t)$ and its derivative $h^{\prime}(t)$ are continuous on the interval $\varphi \leq t \leq \psi$.

iv) As $t$ varies from $\varphi$ to $\psi$, the function $h(t)$ always varies in the same direction from $h(\varphi)=a$ to $h(\psi)=b$.

Let $X_{1: n} \leq X_{2: n} \leq \ldots \leq X_{n: n}$ be the OS obtained by arranging the preceding random sample in increasing order of magnitude. The $c d f$ of $X_{r: n}(1 \leq r \leq n)$ is given by

$$
G_{r: n}(x)=P\left(X_{r: n} \leq x\right)=\sum_{i=r}^{n}\left(\begin{array}{c}
n \\
i
\end{array}\right)[G(x)]^{i}[1-G(x)]^{n-i}=I_{G(x)}(r, n-r+1),
$$

where, the beta function with $B(p, q)=B_{1}(p, q)$, and $I_{x}(p, q)=\frac{B_{x}(p, q)}{B(p, q)}$ is incomplete beta function ratio. The corresponding $p d f$ is given by

$$
\begin{aligned}
g_{r: n}(x)=\frac{\partial}{\partial x}\left[F_{r: n}(x)\right] & =\frac{n !}{(r-1) !(n-r) !}[G(x)]^{r-1}[1-G(x)]^{n-r} g(x) \\
& =g(x) b[G(x) ; r, n-r+1] ;-\infty<x<\infty, 1 \leq r \leq n,
\end{aligned}
$$

where $b(t ; p, q)=\frac{1}{B(p, q)} t^{p-1}(1-t)^{q-1}, 0 \leq t \leq 1$.

Now, consider the random variable, $Y$ as

$Y \sim g_{r: n}(x)$ with probability $\pi_{r: n}(r=1,2,3, \ldots, n ; n=1,2,3, \ldots)$,

where $0 \leq \pi_{r: n} \leq 1$ and $\sum_{r=1}^{n} \pi_{r: n}=1$.

Hence, generalized transmuted $c d f$ of $n$th rank mapped $(n=1,2,3, \ldots)$ distribution is given by

$$
F_{Y}(x)=\sum_{r=1}^{n} \pi_{r: n} I_{G(x)}(r, n-r+1)=\sum_{r=1}^{n} m_{r: n}(x),
$$

and corresponding generalized transmuted $p d f$ of $n$th rank mapped distribution is

$$
\begin{gathered}
f_{Y}(x)=\frac{\partial}{\partial x}\left[F_{Y}(x)\right]=\sum_{r=1}^{n} \frac{\partial}{\partial x}\left[m_{r: n}(x)\right]=\sum_{r=1}^{n} \pi_{r: n} \frac{\partial}{\partial x}\left[I_{G(x)}(r, n-r+1)\right] \\
=g(x) \sum_{r=1}^{n} \pi_{r: n} b[G(x) ; r, n-r+1],
\end{gathered}
$$

where $m_{r: n}(x)=\pi_{r: n} I_{G(x)}(r, n-r+1)$ and $b(. ; .,$.$) is same as before.$

Now,

$$
\begin{aligned}
b(t ; p, q) & =\frac{1}{B(p, q)} t^{p-1}(1-t)^{q-1}=\frac{1}{B(p, q)} t^{p-1} \sum_{i=0}^{q-1}(-1)^{q-1-i}\left(\begin{array}{c}
n \\
i
\end{array}\right) t^{q-1-i} \\
& =\frac{1}{B(p, q)} \sum_{i=0}^{q-1}(-1)^{q-1-i}\left(\begin{array}{c}
n \\
i
\end{array}\right) t^{p+q-2-i}
\end{aligned}
$$


Using (6) in (5) to get

$$
\begin{aligned}
f_{Y}(x) & =g(x) \sum_{r=1}^{n} \sum_{j=0}^{n-r} \frac{(-1)^{n-r-j} \pi_{r: n}}{B(r, n-r+1)}\left(\begin{array}{c}
n-r \\
j
\end{array}\right)[G(x)]^{n-1-j} \\
& =g(x)[G(x)]^{n-1} \sum_{r=1}^{n} \sum_{j=0}^{n-r} k_{r j}(x)=g(x)[G(x)]^{n-1} \sum_{r=1}^{n} k_{r},
\end{aligned}
$$

where, $k_{i}=\sum_{j=0}^{n-i} k_{i j}$ and $k_{i j}=\frac{(-1)^{n-i-j} \pi_{i: n}}{B(i, n-i+1)}\left(\begin{array}{c}n-i \\ j\end{array}\right)[G(x)]^{-j}$.

Expression (3) and (7) will also be helpful for simulation study for the $c d f$ and $p d f$ respectively.

\subsection{Particular Cases}

i) Put $n=2, \pi_{1: n}=\pi$, i.e., $\pi_{2: 2}=1-\pi$ and $\lambda=2 \pi$ in (3) and (7) to get the quadratic transmutation map of Shaw and Buckley [20]

$$
F(x)=\lambda G(x)+(1-\lambda) G^{2}(x)
$$

and the corresponding $p d f$ of quadratic transmutation map of Shaw and Buckley [20] is

$$
f(x)=g(x)[G(x)+\lambda\{1-G(x)\}]
$$

ii) Put $n=3, \pi_{3: 3}=1-\pi_{1: 3}-\pi_{2: 3}$ and $\lambda_{1}=3 \pi_{1: 3}, \lambda_{2}=3 \pi_{2: 3}$ in (3) and (7) to get the cubic ranking transmutation map of Granzotto et al. ([11], Eq. (3), pp. 2761)

$$
F(x)=\lambda_{1} G(x)+\left(\lambda_{2}-\lambda_{1}\right) G^{2}(x)+\left(1-\lambda_{2}\right) G^{3}(x)
$$

and the $p d f$ of cubic ranking transmutation map of Granzotto et al. [11] is

$$
f(x)=g(x)\left[\lambda_{1}+2\left(\lambda_{2}-\lambda_{1}\right) G(x)+3\left(1-\lambda_{2}\right) G^{2}(x)\right] \text {. }
$$

iii) Put $\pi_{1: n}=1$ and $\pi_{i: n}=0 \forall 2 \leq i \leq n$ in (3) and (7) to develop the simple transmutation map of Eugene et al. [6] on using beta distribution as a generator.

$$
F(x)=I_{G(x)}(p, q)
$$

and the pdf of simple transmutation map of Eugene et al. [6] is

$$
f(x)=g(x) b[G(x) ; p, q]
$$

iv) Put $n=3, \lambda=\lambda_{1}=3 \pi_{1: 3}=-\lambda_{2}, \lambda_{2}=3 \pi_{2: 3}$ in (3) and (7) to develop the cubic ranking transmutation map of Al-Kadim and Mohammed [12] with $c d f$

$$
F(x)=(1+\lambda) G(x)-2 \lambda G^{2}(x)-\lambda G^{3}(x)
$$

and the corresponding $p d f$ of the cubic ranking transmutation map of Al-Kadim and Mohammed [12] is

$$
f(x)=(1+\lambda) g(x)-4 \lambda G(x) g(x)-3 \lambda G^{2}(x) g(x) .
$$

It may be noted that the general formula of the transmuted distribution of Al-Kadim [16] can also be deduced from (3) as a particular case for odd and even $n$ respectively. 
v) Put $n=4, \lambda_{i}=2 \pi_{i: 4} ; 1 \leq i \leq 4$ in (3) and (7) to develop the generalized Quartic ranking transmutation map which is new $c d f$ as suggested.

$$
F(x)=2 \lambda_{1} G(x)+3\left(\lambda_{2}-\lambda_{1}\right) G^{2}(x)+2\left(\lambda_{1}-2 \lambda_{2}+\lambda_{3}\right) G^{3}(x)+\left(1-\lambda_{1}+\lambda_{2}-2 \lambda_{3}\right) G^{4}(x)
$$

and the corresponding generalized Quartic ranking transmutation map with new $p d f$ as suggested is given by

$$
f(x)=g(x)\left[2 \lambda_{1}+6\left(\lambda_{2}-\lambda_{1}\right) G(x)+6\left(\lambda_{1}-2 \lambda_{2}+\lambda_{3}\right) G^{2}(x)+4\left(1-\lambda_{1}+\lambda_{2}-2 \lambda_{3}\right) G^{4}(x)\right] .
$$

vi) Put $n=5, \lambda_{i}=5 \pi_{i: 5} ; 1 \leq i \leq 5$ in (3) and (7) to develop the generalized Quintic ranking transmutation map with a new $c d f$ suggested as

$$
\begin{aligned}
F(x)= & \lambda_{1} G(x)+2\left(\lambda_{2}-\lambda_{1}\right) G^{2}(x)+2\left(\lambda_{1}-2 \lambda_{2}+\lambda_{3}\right) G^{3}(x) \\
& +\left(3 \lambda_{2}-\lambda_{1}-3 \lambda_{3}+\lambda_{4}\right) G^{4}(x)+\left(1-\lambda_{2}-\lambda_{3}-\lambda_{4}\right) G^{5}(x)
\end{aligned}
$$

and the corresponding $p d f$ of generalized Quintic ranking transmutation map which is a new $p d f$ as suggested is

$$
\begin{aligned}
f(x)= & g(x)\left[\lambda_{1}+4\left(\lambda_{2}-\lambda_{1}\right) G(x)+6\left(\lambda_{1}-2 \lambda_{2}+\lambda_{3}\right) G^{2}(x)\right. \\
& \left.+4\left(3 \lambda_{2}-\lambda_{1}-3 \lambda_{3}+\lambda_{4}\right) G^{3}(x)+5\left(1-\lambda_{2}-\lambda_{3}-\lambda_{4}\right) G^{4}(x)\right] .
\end{aligned}
$$

vii) Put $\lambda_{i}=n \pi_{i: n} ; 1 \leq i \leq n$, and $n=6,7,8, \ldots$ in (3) and (7) to develop the $c d f$ of suggested generalized desired $n$th ( $\left.n \geq 6\right)$ ranked transmutation map as well as the corresponding $p d f$ of generalized desired $n$th ranked transmutation map.

viii) If one put, $\pi_{i: n}=\frac{1}{n}$ for $1 \leq i \leq n$, then $F(x)=G(x)$

ix) If we put, $\pi_{i: n}=\left(\begin{array}{c}n \\ i\end{array}\right) /\left(2^{n}-1\right)$, for all $1 \leq i \leq n$ and $n=2,3,4, \ldots$, then from (3) and (7) we get another new generalized $n$th ranked transmuted map with $c d f$ for generating families of distributions is

$$
F_{T D n}(x)=\sum_{r=1}^{n} \pi_{r: n} I_{G(x)}(r, n-r+1)=\sum_{r=1}^{n} m_{r: n}(x),
$$

where $m_{r: n}(x)=\left[\left(\begin{array}{c}n \\ r\end{array}\right) /\left(2^{n}-1\right)\right] I_{G(x)}(r, n-r+1)$

and the corresponding generalized $n^{\text {th }}$ ranked transmuted map of $p d f$ for generating families of distribution are given by

$$
f_{T D n}(x)=g(x)[G(x)]^{n-1} \sum_{r=1}^{n} k_{r}
$$

where, $k_{i}=\sum_{j=0}^{n-i} k_{i j}$ and $k_{i j}=\frac{(-1)^{n-i-j}}{\left(2^{n}-1\right) B(i, n-i+1)}\left(\begin{array}{c}n \\ i\end{array}\right)\left(\begin{array}{c}n-i \\ j\end{array}\right)[G(x)]^{-j}$.

\section{Some Specific Cases of (12) and (13)}

\subsubsection{Quadratic rank transmuted distribution (TD2)}

For $n=2$ in (12) and (13), the new form of quadratic map ranked transmuted $c d f$ is given by

$$
F_{T D 2}(x)=\frac{1}{3}\left[4 G(x)-G^{2}(x)\right]=\frac{1}{3} G(x)[3+\{1-G(x)\}]
$$

and the corresponding new form of quadratic map ranked transmuted $p d f$ is given by

$$
f_{T D 2}(x)=\frac{g(x)}{3}[4-2 G(x)]=\frac{2 g(x)}{3}[1+\{1-G(x)\}] .
$$




\subsubsection{Cubic rank transmuted distribution (TD3)}

For $n=3$ in (12) and (13), the new form of cubic ranked transmuted $c d f$ is given by

$$
F_{T D 3}(x)=\frac{1}{7}\left[9 G(x)-2 G^{3}(x)\right]
$$

and the corresponding new form of cubic ranked transmuted $p d f$ is given by

$$
f_{T D 3}(x)=\frac{g(x)}{7}\left[9-6 G^{2}(x)\right] \text {. }
$$

\subsubsection{Quartic rank transmuted distribution (TD4)}

For $n=4$ in (12) and (13), the new form of quartic ranked transmuted $c d f$ is given by

$$
F_{T D 4}(x)=\frac{1}{15}\left[16 G(x)+12 G^{2}(x)-16 G^{3}(x)+3 G^{4}(x)\right]
$$

and the corresponding new form quartic ranked transmuted $p d f$ is given by

$$
f_{T D 4}(x)=\frac{g(x)}{15}\left[16+24 G(x)-48 G^{2}(x)+12 G^{3}(x)\right]
$$

\subsubsection{Quintic rank transmuted distribution (TD5)}

For $n=5$ in (12) and (13), the new form of quintic ranked transmuted $c d f$ is given by

$$
F_{T D 5}(x)=\frac{1}{31}\left[25 G(x)+50 G^{2}(x)-50 G^{3}(x)+6 G^{5}(x)\right]
$$

and the corresponding new form of quintic ranked transmuted $p d f$ is given by

$$
f_{T D 5}(x)=\frac{g(x)}{31}\left[25+100 G(x)-150 G^{2}(x)+30 G^{4}(x)\right] .
$$

In a similar manner, one can generate any desired higher order $(n \geq 6)$ rank transmuted map from (12) and (13).

\section{HAZARD FUNCTION}

The survival function $S(x)$, hazard rate function $h(x)$, and reserved hazard rate function $r(x)$ of newly generated generalized transmuted $c d f F_{T D n}(x)$ (Eq. 12) corresponding to $p d f f_{T D n}(x)$ (13) are respectively given by

$$
\begin{gathered}
S_{T D n}(x)=1-F_{T D n}(x) \\
h_{T D n}(x)=f_{T D n}(x) / S_{T D n}(x)=f_{T D n}(x) /\left\{1-F_{T D n}(x)\right\} \\
r_{T D n}(x)=f_{T D n}(x) / F_{T D n}(x),
\end{gathered}
$$

where, $f_{T D n}(x)$ and $F_{T D n}(x)$ are as before.

Use (3) in (22) to get survival function for newly derived $n$th ranked transmuted distribution as

$$
S_{T D n}(x)=1-\sum_{r=1}^{n} m_{r: n}(x) .
$$

Use (7) and (22) in (23) to get hazard rate function for newly derived $n$th ranked transmuted distribution as

$$
h_{T D n}(x)=\frac{g(x)[G(x)]^{n-1} \sum_{r=1}^{n} k_{r}}{1-\sum_{r=1}^{n} m_{r: n}(x)} .
$$


Use (3) and (7) in (24) to get reserved hazard rate function for newly derived $n$th ranked transmuted distribution as

$$
r_{T D n}(x)=\frac{g(x)[G(x)]^{n-1} \sum_{r=1}^{n} k_{r}}{\sum_{r=1}^{n} m_{r: n}(x)},
$$

where, $m_{r: n}(x)$ and $k_{r}$ are as before.

For more about the hazard function one can refer to Zubair et al. [21] and references therein.

Theorem 3.1. The quadratic generalized transmuted hazard rate function is given by

$$
h_{T D 2}(x)=\frac{2 g(x)[2-G(x)]}{3-G(x)[4-G(x)]} .
$$

Proof. From (23) and for $n=2$, we get the generalized transmuted hazard rate function as

$$
h_{T D 2}(x)=\frac{f_{T D 2}(x)}{1-F_{T D 2}(x)} .
$$

Now using (14) and (15) in (28) and on algebraic simplification gives the proof of the theorem.

Theorem 3.2. The quadratic generalized transmuted Weibull hazard rate function is given by

$$
h_{T W D 2}(x)=\frac{2 \alpha x^{\alpha-1}\left[e^{-(x / \beta)^{\alpha}}+e^{-(x / \beta)^{2 \alpha}}\right]}{3 \beta^{\alpha}\left[1-\frac{2}{3} e^{-(x / \beta)^{\alpha}}+\frac{1}{3} e^{-(x / \beta)^{2 \alpha}}\right]} .
$$

Proof. From (28) and on using (29) and (30) gives the proof of the theorem.

Simulated hazard function (WD, TWD2 to TWD5) for some specific sets of parameters $(\alpha, \beta)$ values are presented in Figures 1-4 to compare among themselves. The hazard function is one of the most important quantities to character life phenomenon. Compare with many other modified Weibull distributions, the shape of the hazard function is easy to decide. It can be derived from (23) and it is flexible. As we know, it is very common for a bathtub-shaped hazard function of a system or component to have a long useful lifetime with low constant rate portion in the middle and sharp change in the initial and wear-out of phase, so a distribution which can fit this kind of hazard rate would be very useful in reliability studies.

\section{TRANSMUTED WEIBULL DISTRIBUTION}

The Weibull distribution which was proposed by Weibull [22] is a very important lifetime distribution and is widely used in many fields. However, the hazard function of the traditional Weibull distribution can only be increasing, decreasing, or constant. To meet the need of fitting complex modes and the bathtub-shaped hazard rate, researchers have proposed many improved flexible models based on the traditional Weibull distribution. To know more about modified or improved models based on the traditional Weibull distribution, one may refer to Johnson et al. [5], Xie et al. [23], Bebbington et al. [24], Nassar et al. [25], Afify et al. [19], and references therein. Still even available modified Weibull model are not enough to represent or fit the data obtain all cases such as engineering, economics, finance and actuarial

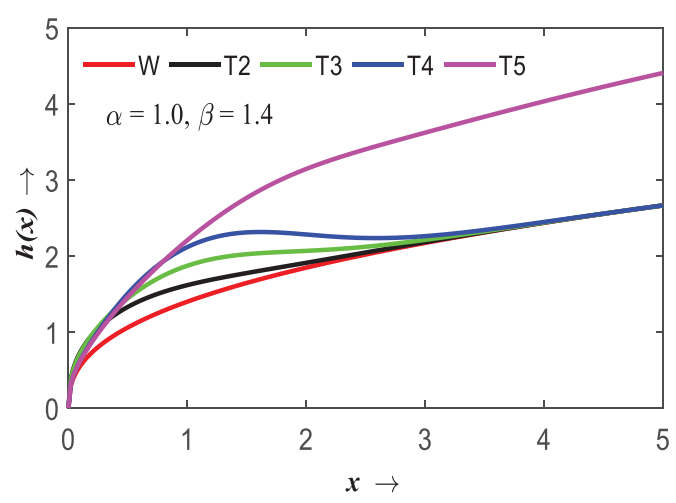

Figure 1 Hazard rate curve for $\alpha=1.0, \beta=1.4$. 


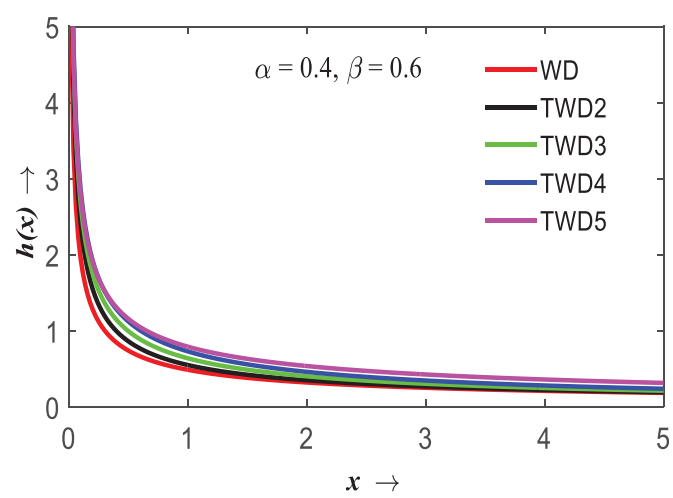

Figure 2 Hazard rate curve for $\alpha=0.4, \beta=0.6$.

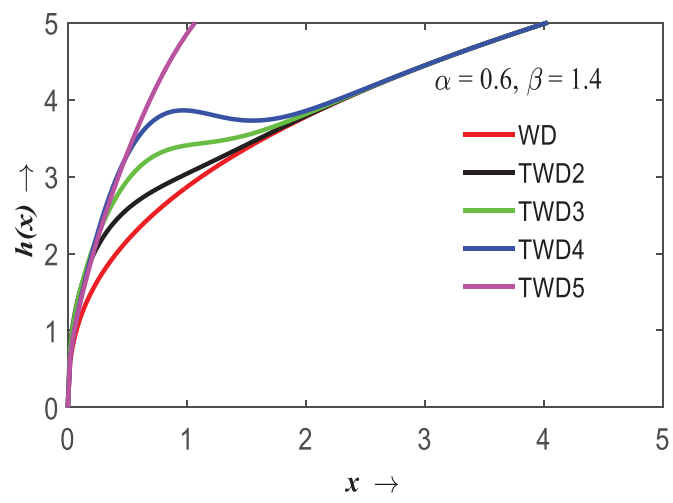

Figure 3 Hazard rate curve for $\alpha=0.6, \beta=1.4$.

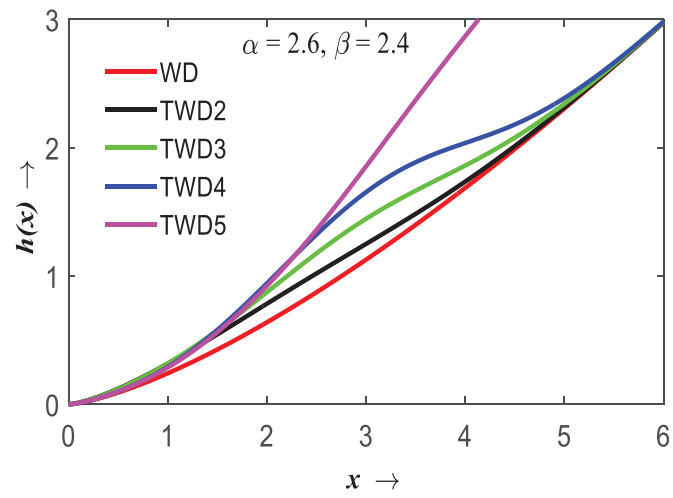

Figure 4 Hazard rate curve for $\alpha=2.6, \beta=2.4$.

science, medical and life sciences. Our proposed transmuted model will be more flexible and will cover such limitation for which data present a higher degree of skewness and kurtosis.

A random variable $X$ is said to have traditional Weibull distribution (WD) with parameters $(\alpha>0, \beta>0, \theta \geq 0)$ if its $c d f$ is given by

$$
G(x)=1-\exp \left\{-\left(\frac{x-\theta}{\beta}\right)^{\alpha}\right\} ; x \geq \theta
$$

and corresponding $p d f$ is given by

$$
g(x)=\frac{\alpha}{\beta}\left(\frac{x-\theta}{\beta}\right)^{\alpha-1} \exp \left\{-\left(\frac{x-\theta}{\beta}\right)^{\alpha}\right\} ; x \geq \theta,
$$

where, $\alpha$ is the shape parameter, $\beta$ is the scale parameter, and $\theta$ is the location parameter. Throughout the paper take $\theta=0$ without loss of generality, then (29) and (30) transform into $c d f$ and $p d f$ of 2-parameters traditional Weibull distribution, respectively. 
i) Quadratic ranked map Transmuted Weibull distribution (TWD2)

Using (29) in (14); (29) and (30) into (15) to get the new Quadratic map ranked transmuted Weibull $c d f$ as

$$
F_{T W D 2}(x)=\left[1-\exp \left\{-\left(\frac{x}{\beta}\right)^{\alpha}\right\}\right]\left[1+\frac{1}{3} \exp \left\{-\left(\frac{x}{\beta}\right)^{\alpha}\right\}\right]
$$

and the corresponding new Quadratic map ranked transmuted Weibull $p d f$ is given by

$$
f_{T W D 2}(x)=\frac{2 \alpha}{3 \beta}\left(\frac{x}{\beta}\right)^{\alpha-1} \exp \left\{-\left(\frac{x}{\beta}\right)^{\alpha}\right\}\left[1+\exp \left\{-\left(\frac{x}{\beta}\right)^{\alpha}\right\}\right]
$$

ii) Cubic ranked map Transmuted Weibull distribution (TWD3)

Using (29) in to (16); (29) and (30) into (17) to get the new Cubic map ranked transmuted Weibull cdf as

$$
F_{T W D 3}(x)=\frac{1}{7}\left[9\left[1-\exp \left\{-\left(\frac{x}{\beta}\right)^{\alpha}\right\}\right]-2\left[1-\exp \left\{-\left(\frac{x}{\beta}\right)^{\alpha}\right\}\right]^{3}\right]
$$

and the corresponding new cubic map ranked transmuted Weibull $p d f$ is given by

$$
f_{T W D 3}(x)=\frac{\alpha}{7 \beta}\left(\frac{x}{\beta}\right)^{\alpha-1} \exp \left\{-\left(\frac{x}{\beta}\right)^{\alpha}\right\}\left[9-6\left(1-\exp \left\{-\left(\frac{x}{\beta}\right)^{\alpha}\right\}\right)^{2}\right]
$$

iii) Quartic ranked map Transmuted Weibull distribution (TWD4)

Using (29) in to (18); (29) and (30) into (19) to get the new Quartic map ranked transmuted Weibull $c d f$ as

$$
\begin{aligned}
F_{T W D 4}(x)= & \frac{1}{15}\left[16\left\{1-\exp \left\{-\left(\frac{x}{\beta}\right)^{\alpha}\right\}\right\}+12\left\{1-\exp \left\{-\left(\frac{x}{\beta}\right)^{\alpha}\right\}\right\}^{2}\right. \\
& \left.-16\left\{1-\exp \left\{-\left(\frac{x}{\beta}\right)^{\alpha}\right\}\right\}^{3}+3\left\{1-\exp \left\{-\left(\frac{x}{\beta}\right)^{\alpha}\right\}\right\}^{4}\right]
\end{aligned}
$$

and the corresponding new Quartic ranked transmuted Weibull $p d f$ is given by

$$
\begin{aligned}
f_{T W D 4}(x)= & \frac{\alpha}{15 \beta}\left(\frac{x}{\beta}\right)^{\alpha-1} \exp \left\{-\left(\frac{x}{\beta}\right)^{\alpha}\right\}\left[16+24\left\{1-\exp \left\{-\left(\frac{x}{\beta}\right)^{\alpha}\right\}\right\}\right. \\
& \left.-48\left\{1-\exp \left\{-\left(\frac{x}{\beta}\right)^{\alpha}\right\}\right\}^{2}+12\left\{1-\exp \left\{-\left(\frac{x}{\beta}\right)^{\alpha}\right\}\right\}^{3}\right] .
\end{aligned}
$$

iv) Quintic ranked map Transmuted Weibull distribution (TWD5)

Using (29) in to (20); (29) and (30) into (21) to get new the Quartic map ranked transmuted Weibull $c d f$ as

$$
\begin{aligned}
F_{T W D 5}(x)= & \frac{1}{31}\left[25\left\{1-\exp \left\{-\left(\frac{x}{\beta}\right)^{\alpha}\right\}\right\}+50\left\{1-\exp \left\{-\left(\frac{x}{\beta}\right)^{\alpha}\right\}\right\}^{2}\right. \\
& \left.-50\left\{1-\exp \left\{-\left(\frac{x}{\beta}\right)^{\alpha}\right\}\right\}^{3}+6\left\{1-\exp \left\{-\left(\frac{x}{\beta}\right)^{\alpha}\right\}\right\}^{5}\right]
\end{aligned}
$$

and the corresponding new quartic ranked transmuted Weibull $p d f$ is given by

$$
\begin{aligned}
f_{\text {TWD } 5}(x)= & \frac{\alpha}{15 \beta}\left(\frac{x}{\beta}\right)^{\alpha-1} \exp \left\{-\left(\frac{x}{\beta}\right)^{\alpha}\right\}\left[25+100\left\{1-\exp \left\{-\left(\frac{x}{\beta}\right)^{\alpha}\right\}\right\}\right. \\
& \left.-150\left\{1-\exp \left\{-\left(\frac{x}{\beta}\right)^{\alpha}\right\}\right\}^{2}+18\left\{1-\exp \left\{-\left(\frac{x}{\beta}\right)^{\alpha}\right\}\right\}^{4}\right] .
\end{aligned}
$$




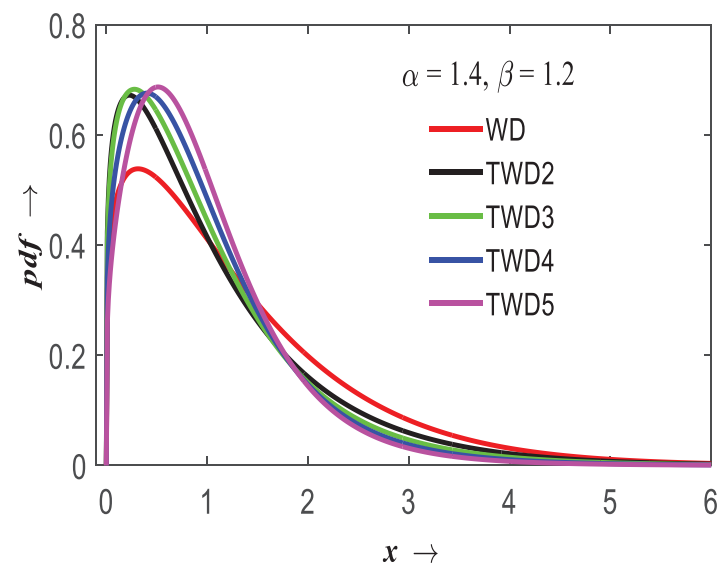

Figure 5 TWDn curve for $\alpha=1.4, \beta=1.2$.

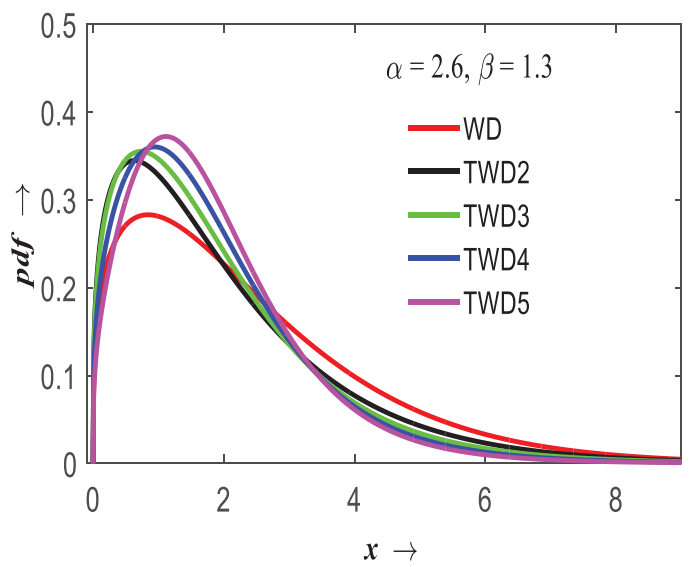

Figure 6 TWDn curve for $\alpha=2.6, \beta=1.3$.

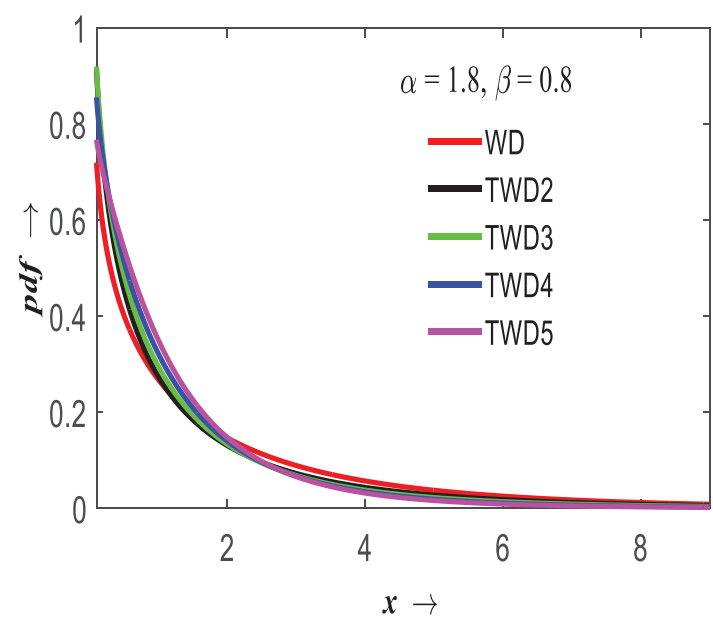

Figure 7 TWDn curve for $\alpha=1.8, \beta=0.8$.

In a similar way one can find new any desired rank map transmuted $c d f$ of Weibull distribution by using (29) in to (12); corresponding $p d f$ by using (29) and (30) into (13).

The simulated $p d f$ curves of different ranked TWD for some specific sets of parametric values $(\alpha, \beta)$ were plotted in Figures $5-8$ to observe and compare the change of skewness and the $p d f$ curve shapes with the change of transmutation rank.

It is observed from the above Figures 5-8 that the TWDs are more skewed compare to ordinary Weibull distribution. The degree of skewness of TWDn curves increases if the rank of transmutation map increases. So, the newly generated TWDs have advantages to fit if the data sets are more skewed. 


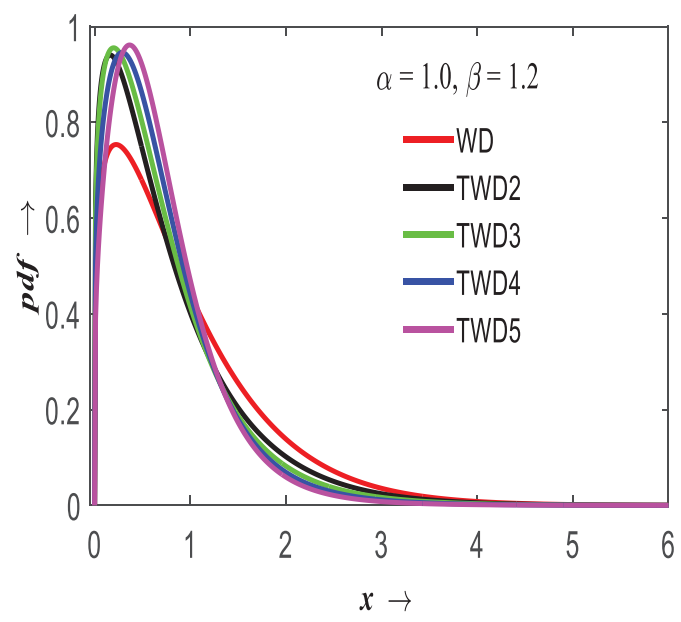

Figure 8 TWDn curve for $\alpha=1.0, \beta=1.2$.

\section{DISTRIBUTIONAL CHARACTERISTICS}

The $k^{\text {th }}(k=1,2,3, \ldots)$ raw moment corresponding to generalized $n^{\text {th }}$ rank transmuted map of Weibull pdf (30) are given from (13) as

$$
\mu_{k}^{\prime}=E\left(X^{k}\right)=\int_{0}^{\infty} x^{k} f(x) d x=\sum_{r=1}^{n} k_{r}
$$

where, $k_{i}=\sum_{j=0}^{n-i} k_{i j}, k_{i j}=\frac{(-1)^{n-i-j}}{\left(2^{n}-1\right) B(i, n-i+1)}\left(\begin{array}{c}n \\ i\end{array}\right)\left(\begin{array}{c}n-i \\ j\end{array}\right) M_{k, n-1-j}$

and $M_{k, m}=\int_{0}^{\infty} x^{k} g(x) G^{m}(x) d x$.

Now on using (29) and (30), we have

$$
\begin{aligned}
M_{k, m} & =\frac{\alpha}{\beta} \int_{0}^{\infty} x^{k}\left(\frac{x}{\beta}\right)^{\alpha-1} \exp \left\{-\left(\frac{x}{\beta}\right)^{\alpha}\right\}\left[1-\exp \left\{-\left(\frac{x}{\beta}\right)^{\alpha}\right\}\right]^{m} d x \\
& =\frac{\alpha}{\beta^{\alpha}} \int_{0}^{\infty} x^{k+\alpha-1} e^{-\left(\frac{x}{\beta}\right)^{\alpha}} \sum_{i=0}^{m}(-1)^{i}\left(\begin{array}{c}
m \\
i
\end{array}\right) \exp \left\{-\left(\frac{x}{\beta}\right)^{\alpha i}\right\} d x \\
& =\frac{\alpha}{\beta^{\alpha}} \sum_{i=0}^{m}(-1)^{i}\left(\begin{array}{c}
m \\
i
\end{array}\right) \int_{0}^{\infty} x^{k+\alpha-1} \exp \left\{-\left(\frac{x}{\beta}\right)^{\alpha i+1}\right\} d x .
\end{aligned}
$$

We know that $\int_{0}^{\infty} x^{n} \exp \left\{-(q x)^{m}\right\} d x=\frac{\Gamma\left(\frac{n+1}{m}\right)}{m q^{n+1}}, m, n>0$ and real. For more about it refer to Jeffrey and Dai ([26], p. 272). So that

$$
M_{k, m}=\alpha \beta^{k} \sum_{m}^{m}(-1)^{i}\left(\begin{array}{c}
m \\
i
\end{array}\right) \frac{\Gamma\left(\frac{k+\alpha}{\alpha i+1}\right)}{(\alpha i+1)}=\alpha \beta^{k} \sum_{i=0}^{m} \delta_{i m},
$$

where, $\delta_{i m}=(-1)^{i}\left(\begin{array}{c}m \\ i\end{array}\right) \frac{\Gamma\left(\frac{k+\alpha}{\alpha i+1}\right)}{(\alpha i+1)}$.

\subsection{Moments for Quadratic TWD2}

For $n=2$ in (39), the $k$ th raw moment of the quadratic TWD is given by

$$
\mu_{k}^{\prime}=E\left(X^{k}\right)=\int_{0}^{\infty} x^{k} f(x) d x=\frac{\beta^{k}}{3}\left[2 \Gamma\left(\frac{k+\alpha}{\alpha}\right)+\Gamma\left(\frac{k+\alpha}{2 \alpha}\right)\right]
$$


and for $k=1,2,3,4$, the first four moments of the quadratic TWD are given by

$$
\begin{gathered}
\text { Mean }=\mu_{1}^{\prime}=\frac{\beta}{3}\left[2 \Gamma\left(\frac{1+\alpha}{\alpha}\right)+\Gamma\left(\frac{1+\alpha}{2 \alpha}\right)\right] \\
\mu_{2}^{\prime}=\frac{\beta^{2}}{3}\left[2 \Gamma\left(\frac{2+\alpha}{\alpha}\right)+\Gamma\left(\frac{2+\alpha}{2 \alpha}\right)\right] \\
\mu_{3}^{\prime}=\frac{\beta^{3}}{3}\left[2 \Gamma\left(\frac{3+\alpha}{\alpha}\right)+\Gamma\left(\frac{3+\alpha}{2 \alpha}\right)\right] \\
\text { and } \mu_{4}^{\prime}=\frac{\beta^{4}}{3}\left[2 \Gamma\left(\frac{4+\alpha}{\alpha}\right)+\Gamma\left(\frac{4+\alpha}{2 \alpha}\right)\right] .
\end{gathered}
$$

The central moments are given by

$$
\begin{aligned}
\operatorname{Var}(X) & =\mu_{2}=\frac{\beta^{2}}{3}\left[2 \Gamma\left(\frac{2+\alpha}{\alpha}\right)+\Gamma\left(\frac{2+\alpha}{2 \alpha}\right)\right]-\frac{\beta^{2}}{9}\left[2 \Gamma\left(\frac{1+\alpha}{\alpha}\right)+\Gamma\left(\frac{1+\alpha}{2 \alpha}\right)\right]^{2} \\
\mu_{3} & =\mu_{3}^{\prime}-3 \mu_{2}^{\prime} \mu_{1}^{\prime}+2\left(\mu_{1}^{\prime}\right)^{3} \\
\text { and } \mu_{4} & =\mu_{4}^{\prime}-4 \mu_{3}^{\prime} \mu_{1}^{\prime}+6 \mu_{2}^{\prime}\left(\mu_{1}^{\prime}\right)^{2}-3\left(\mu^{\prime}\right)^{4} .
\end{aligned}
$$

Pearson's four coefficients, based upon the first four central moments are

$$
\beta_{1}=\frac{\mu_{3}^{2}}{\mu_{2}^{3}}, \gamma_{1}=+\sqrt{\beta_{1}} \text { and } \beta_{2}=\frac{\mu_{4}}{\mu_{2}^{2}}, \gamma_{2}=\beta_{2}-3
$$

It may be noted that these coefficients are true numbers independent of units of measurement.

The $p \operatorname{th}[p \in(0,1)]$ percentile point of quadratic TWD (32) is given by

$$
x_{p}=\beta[-\ln \{1-2(1-\sqrt{1-3 p / 4})\}]^{1 / \alpha}
$$

and random observation can be generated from the following inverse function

$X=\beta[-\ln \{1-2(1-\sqrt{1-3 U / 4})\}]^{1 / \alpha}$, where $U \sim U(0,1)$.

\subsection{Moments for Cubic TWD3}

For $n=3$ in (39), the $k$ th raw moment of the Cubic TWD is given by

$$
\mu_{k}^{\prime}=E\left(X^{k}\right)=\sum_{r=1}^{3} k_{r}=k_{1}+k_{2}+k_{3}
$$

where, $k_{1}=\sum_{j=0}^{2} k_{1 j}=k_{10}+k_{11}+k_{12}, k_{2}=\sum_{j=0}^{1} k_{1 j}=k_{20}+k_{21}, k_{3}=k_{30}$.

$k_{10}=\frac{9}{7} M_{k, 2}, k_{11}=\frac{18}{7} M_{k, 1}, k_{12}=\frac{9}{7} M_{k, 0}, k_{20}=-\frac{18}{7} M_{k, 2}, k_{21}=\frac{18}{7} M_{k, 1}, k_{30}=\frac{3}{7} M_{k, 2}$.

$M_{k, 0}=\alpha \beta^{k} \delta_{00}, M_{k, 1}=\alpha \beta^{k}\left(\delta_{01}+\delta_{11}\right), M_{k, 2}=\alpha \beta^{k}\left(\delta_{02}+\delta_{12}+\delta_{22}\right)$.

$\delta_{00}=\Gamma(k+\alpha), \delta_{01}=\Gamma(k+\alpha), \delta_{02}=\Gamma(k+\alpha), \delta_{11}=-\frac{1}{1+\alpha} \Gamma\left(\frac{k+\alpha}{1+\alpha}\right), \delta_{12}=-\frac{2}{1+\alpha} \Gamma\left(\frac{k+\alpha}{1+\alpha}\right), \delta_{22}=-\frac{1}{1+2 \alpha} \Gamma\left(\frac{k+\alpha}{1+2 \alpha}\right)$.

From (34), the $r^{\text {th }}$ raw moment of TWD3 is given by

$$
\mu_{k}^{\prime}=E(X)^{k}=\frac{\beta^{k}}{7}\left[3 \Gamma\left(\frac{\alpha+k}{\alpha}\right)+6 \Gamma\left(\frac{\alpha+k}{2 \alpha}\right)-2 \Gamma\left(\frac{\alpha+k}{3 \alpha}\right)\right] .
$$




$$
\begin{aligned}
& \text { Mean }=\mu_{1}^{\prime}=\frac{\beta}{7}\left[3 \Gamma\left(\frac{\alpha+1}{\alpha}\right)+6 \Gamma\left(\frac{\alpha+1}{2 \alpha}\right)-2 \Gamma\left(\frac{\alpha+1}{3 \alpha}\right)\right] \text {, } \\
& \mu_{2}^{\prime}=\frac{\beta^{2}}{7}\left[3 \Gamma\left(\frac{\alpha+2}{\alpha}\right)+6 \Gamma\left(\frac{\alpha+2}{2 \alpha}\right)-2 \Gamma\left(\frac{\alpha+2}{3 \alpha}\right)\right] \\
& \mu_{3}^{\prime}=\frac{\beta^{3}}{7}\left[3 \Gamma\left(\frac{\alpha+3}{\alpha}\right)+6 \Gamma\left(\frac{\alpha+3}{2 \alpha}\right)-2 \Gamma\left(\frac{\alpha+3}{3 \alpha}\right)\right], \\
& \mu_{4}^{\prime}=\frac{\beta^{4}}{7}\left[3 \Gamma\left(\frac{\alpha+4}{\alpha}\right)+6 \Gamma\left(\frac{\alpha+4}{2 \alpha}\right)-2 \Gamma\left(\frac{\alpha+4}{3 \alpha}\right)\right] . \\
& \text { and } \operatorname{Var}(X)=\frac{\beta^{2}}{7}\left[3 \Gamma\left(\frac{\alpha+2}{\alpha}\right)+6 \Gamma\left(\frac{\alpha+2}{2 \alpha}\right)-2 \Gamma\left(\frac{\alpha+2}{3 \alpha}\right)\right] \\
& -\frac{\beta^{2}}{49}\left[3 \Gamma\left(\frac{\alpha+1}{\alpha}\right)+6 \Gamma\left(\frac{\alpha+1}{2 \alpha}\right)-2 \Gamma\left(\frac{\alpha+1}{3 \alpha}\right)\right]^{2} \text {. }
\end{aligned}
$$

Other central moments and Pearson's four coefficients can be obtained from the above by simple algebraic manipulation.

\subsection{Moments for Quartic TWD4}

For $n=4$ in (39), the $k$ th raw moment of the TWD4 is given by

$$
\mu_{k}^{\prime}=E(X)^{k}=\frac{\beta^{k}}{15}\left[4 \Gamma\left(\frac{\alpha+k}{\alpha}\right)+18 \Gamma\left(\frac{\alpha+k}{2 \alpha}\right)-4 \Gamma\left(\frac{\alpha+k}{3 \alpha}\right)-3 \Gamma\left(\frac{\alpha+k}{4 \alpha}\right)\right]
$$

\subsection{Moments for Quintic TWD5}

For $n=5$ in (39), the $k$ th raw moment of the TWD5 is given by

$$
\begin{aligned}
\mu_{k}^{\prime}= & E\left(X^{k}\right)=\frac{\beta^{k}}{31}\left[5 \Gamma\left(\frac{\alpha+k}{\alpha}\right)+40 \Gamma\left(\frac{\alpha+k}{2 \alpha}\right)+10 \Gamma\left(\frac{\alpha+k}{3 \alpha}\right)\right. \\
& \left.-30 \Gamma\left(\frac{\alpha+k}{4 \alpha}\right)+6 \Gamma\left(\frac{\alpha+k}{5 \alpha}\right)\right] .
\end{aligned}
$$

\begin{tabular}{|c|c|c|c|c|c|c|c|c|c|c|c|}
\hline \multirow{2}{*}{\multicolumn{2}{|c|}{$\begin{array}{l}\text { Distributional } \\
\text { Characteristics }\end{array}$}} & \multicolumn{9}{|c|}{ Different Combination of Parameter $(\alpha, \beta)$ Values } & \multirow{2}{*}{$\begin{array}{l}\alpha=0.5 \\
\beta=0.1\end{array}$} \\
\hline & & $\alpha=0.4$ & $\alpha=1.0$ & $\alpha=1.3$ & $\alpha=1.4$ & $\alpha=1.5$ & $\alpha=1.5$ & $\alpha=0.5$ & $\alpha=2.0$ & $\alpha=0.6$ & \\
\hline \multirow{6}{*}{ Means } & WD & 0.9970 & 1.0000 & 1.1083 & 1.0937 & 1.1736 & 1.0833 & 0.6000 & 0.2659 & 1.9559 & 0.2000 \\
\hline & TWD2 & 0.7566 & 1.0000 & 1.1714 & 1.1714 & 1.2715 & 1.1737 & 0.4886 & 0.2998 & 1.6909 & 0.1629 \\
\hline & TWD3 & 0.5841 & 0.8988 & 1.0684 & 1.0717 & 1.1662 & 1.0765 & 0.3993 & 0.2771 & 1.4330 & 0.1331 \\
\hline & TWD4 & 0.4571 & 0.7511 & 0.8879 & 0.8890 & 0.9658 & 0.8915 & 0.3255 & 0.2280 & 1.1889 & 0.1085 \\
\hline & TWD5 & 0.3654 & 0.6025 & 0.6980 & 0.6951 & 0.7515 & 0.6936 & 0.2673 & 0.1743 & 0.9806 & 0.0891 \\
\hline & WD & 9.8060 & 1.0000 & 0.7391 & 0.6266 & 0.6349 & 0.5410 & 1.8000 & 0.0193 & 11.8246 & $\begin{array}{l}0.2000 \\
\text { (Continued) }\end{array}$ \\
\hline
\end{tabular}

For example, some distributional properties like mean, variance, skewness, and kurtosis are simulated and presented below in Table 1 for some specific values of the parameters $(\alpha, \beta)$ to observe and compare differentiation of traditional Weibull distribution (30) along with some other different ranked map TWD (TWD $n, n=2,3,4,5)$, where, $n$ indicates $n$th rank (31-38) map. It is observed that skewness of transmuted distribution is more flexible as rank of transmutation increases. So, one can use flexible desired rank map transmuted distribution to fit desired skewed data set. For all simulation work MATLAB R2015a version is used.

Table 1 Distributional characteristics of $n^{\text {th }}, n=2,3,4, \ldots$ degree mapof transmuted Weibull distribution (TWD $\left.n, n=2,3,4, \ldots\right)$. 
Table 1 Distributional characteristics of $n^{\text {th }}, n=2,3,4, \ldots$ degree mapof transmuted Weibull distribution (TWD $\left.n, n=2,3,4, \ldots\right)$. (Continued)

\begin{tabular}{|c|c|c|c|c|c|c|c|c|c|c|c|}
\hline \multirow{2}{*}{\multicolumn{2}{|c|}{$\begin{array}{l}\text { Distributional } \\
\text { Characteristics }\end{array}$}} & \multicolumn{9}{|c|}{ Different Combination of Parameter $(\alpha, \beta)$ Values } & \multirow{2}{*}{$\begin{aligned} \alpha & =0.5 \\
\beta & =0.1\end{aligned}$} \\
\hline & & $\alpha=0.4$ & $\alpha=1.0$ & $\alpha=1.3$ & $\alpha=1.4$ & $\alpha=1.5$ & $\alpha=1.5$ & $\alpha=0.5$ & $\alpha=2.0$ & $\alpha=0.6$ & \\
\hline \multirow{4}{*}{ Variances } & TWD2 & 6.6876 & 0.6287 & 0.3727 & 0.2819 & 0.2473 & 0.2107 & 1.2411 & 0.0001 & 8.1840 & 0.1379 \\
\hline & TWD3 & 4.4160 & 0.5232 & 0.3566 & 0.2867 & 0.2715 & 0.2314 & 0.8456 & 0.0041 & 5.7940 & 0.0940 \\
\hline & TWD4 & 2.8471 & 0.5209 & 0.4601 & 0.4087 & 0.4322 & 0.3683 & 0.5756 & 0.0156 & 4.2317 & 0.0640 \\
\hline & TWD5 & 1.7331 & 0.3269 & 0.2397 & 0.1943 & 0.1842 & 0.1570 & 0.3615 & 0.0022 & 2.7453 & 0.0402 \\
\hline \multirow{5}{*}{ Skewness } & WD & 142644.2686 & 35.0000 & 21.4433 & 15.4247 & 19.0513 & 11.7856 & 372.0816 & 0.0013 & 67852.7372 & 0.5104 \\
\hline & TWD2 & 63555.1105 & 18.5292 & 13.1605 & 9.9132 & 12.7894 & 7.9119 & 166.8259 & 0.0010 & 30860.4307 & 0.2288 \\
\hline & TWD3 & 26345.0289 & 9.9275 & 8.1805 & 6.4000 & 8.5316 & 5.2779 & 69.9412 & 0.0008 & 13291.0082 & 0.0959 \\
\hline & TWD4 & 10238.2132 & 5.4371 & 5.1186 & 4.1148 & 5.6021 & 3.4656 & 27.6680 & 0.0005 & 5489.6068 & 0.0380 \\
\hline & TWD5 & 3764.5469 & 3.2185 & 3.4063 & 2.7832 & 3.8259 & 2.3668 & 10.4650 & 0.0004 & 2226.7486 & 0.0144 \\
\hline \multirow{5}{*}{ Kurtosis } & WD & 305.6793 & 24.0000 & 25.1054 & 26.5616 & 28.4265 & 28.4265 & 100.8000 & 43.4273 & 52.9786 & 100.8000 \\
\hline & TWD2 & 438.1475 & 41.5948 & 70.8843 & 95.9613 & 139.5531 & 139.5531 & 141.3656 & 741.4459 & 73.7997 & 141.3656 \\
\hline & TWD3 & 645.9979 & 40.8002 & 56.3043 & 68.9036 & 87.7369 & 87.7369 & 195.8565 & 642.8510 & 94.8898 & 195.8565 \\
\hline & TWD4 & 967.0436 & 27.9066 & 24.9716 & 25.6054 & 26.6692 & 26.6692 & 263.1634 & 36.1091 & 111.1517 & 263.1634 \\
\hline & TWD5 & 1578.6245 & 48.6008 & 69.6797 & 87.7467 & 115.7325 & 115.7325 & 404.0340 & 455.9642 & 160.7934 & 404.0340 \\
\hline
\end{tabular}

\section{ORDER STATISTICS}

OS and functions of OS play an important role in statistical theory and methodology. Floods and droughts, longevity, breaking strength, aeronautics, oceanography, duration of humans, organisms, components, and devices of various kinds can be studied by the theory extreme values. Life tests provide an ideal illustration of the advantage of OS in censored data. Since such an experiment may take a long time to complete, it is often advantageous to stop after failure of the first $r$ out of $n$ similar items under test. For more details and development of OS one may refer to Sarhan and Greenberg [27], Arnold and Balakrishnan [28], Balakrishnan and Cohen [29], Arnold et al. [30], Ali [31], and David and Nagaraja [32].

The $p d f$ of $r^{\text {th }}$ OS for the TD2 (15) is given by

$$
\left(f_{r: n}\right)_{T D 2}=B(r, n-r+1)\left[F_{T D 2}(x)\right]^{r-1}\left[1-F_{T D 2}(x)\right]^{n-r} f_{T D 2}(x) .
$$

The $p d f$ of extreme OS follows from (45) at $r=1$ and $r=n$ respectively given by

$$
\begin{gathered}
\left(f_{1: n}\right)_{T D 2}=n\left[1-F_{T D 2}(x)\right]^{n-1} f_{T D 2}(x) . \\
\left(f_{n: n}\right)_{T D 2}=n\left[F_{T D 2}(x)\right]^{n-1} f_{T D 2}(x) .
\end{gathered}
$$

Theorem 6.1. For $n=2,3, \ldots$, the recurrence relation between the $p d f$ of largest OS of quadratic rank transmuted distribution given in (47) and any ordinary distribution is given by

$$
\left(f_{n: n}\right)_{T D 2}(x)=n(4 / 3)^{n} \sum_{i=0}^{n-1}(-1 / 4)^{i}\left(\begin{array}{c}
n-1 \\
i
\end{array}\right)\left[\frac{g_{n+i: n+i}}{n+i}-\frac{g_{n+i+1: n+i+1}}{2(n+i+1)}\right]
$$

where, $g_{m: m}=m G^{m-1}(x) g(x) . G(x)$ and $g(x)$ are $c d f$ and $p d f$ of any continuous distribution, respectively.

Proof. From (47) and on using (14) and (15), we have

$$
\begin{aligned}
\left(f_{n: n}\right)_{T D 2}(x) & =n\left[\frac{1}{3}\left\{4 G(x)-G^{2}(x)\right\}\right]^{n-1} \frac{g(x)}{3}[4-2 G(x)] \\
& =n(4 / 3)^{n} G^{n-1}(x)\left[1-\frac{1}{4} G(x)\right]^{n-1}\left[1-\frac{1}{2} G(x)\right] g(x) \\
& =n(4 / 3)^{n} \sum_{i=0}^{n-1}(-1 / 4)^{i}\left(\begin{array}{c}
n-1 \\
i
\end{array}\right)\left[G^{n+i-1}(x)-\frac{1}{2} G^{n+i}(x)\right] g(x) .
\end{aligned}
$$


Now using $g_{m: m}=m G^{m-1}(x) g(x)$ to get the required result.

Theorem 6.2. For $n=2,3, \ldots$, the recurrence relation between $k t h$ order moment of largest OS for the pdf (47) of quadratic rank transmuted distribution and $k t h$ order moment of largest OS for the $p d f$ of ordinary continuous distribution is given by

$$
\left(\mu_{n: n}^{(k)}\right)_{T D 2}=n(4 / 3)^{n} \sum_{i=0}^{n-1}(-1 / 4)^{i}\left(\begin{array}{c}
n-1 \\
i
\end{array}\right)\left[\frac{\mu_{n+i: n+i}^{(k)}}{n+i}-\frac{\mu_{n+i+1: n+i+1}^{(k)}}{2(n+i+1)}\right]
$$

Proof. Multiplying both sides of (48) by $X^{k}$ and then take expectation to get the result of the theorem.

Theorem 6.3. For $n=2,3, \ldots$, the recurrence relation between largest OS $p d f$ of (47) of quadratic rank transmuted distribution and largest OS pdf of Weibull distribution (30) is given by

$$
\begin{aligned}
\left(f_{n: n}\right)_{T W D 2}(x)= & n\left(\frac{4}{3}\right)^{n} \frac{\alpha}{\beta}\left(\frac{x}{\beta}\right)^{\alpha-1}\left[\sum _ { i = 0 } ^ { n - 1 } \sum _ { j = 0 } ^ { n + i - 1 } \frac { ( - 1 ) ^ { i + j } } { 4 ^ { i } } ( \begin{array} { c } 
{ n - 1 } \\
{ i }
\end{array} ) \left\{\left(\begin{array}{c}
n+i-1 \\
j
\end{array}\right)\right.\right. \\
& \left.\left.-\frac{1}{2}\left(\begin{array}{c}
n+i \\
j
\end{array}\right)\right\} e^{-(x / \beta)^{(j+1) \alpha}}-\sum_{i=0}^{n-1} \frac{(-1)^{n+2 i}}{4^{i}} \frac{1}{2}\left(\begin{array}{c}
n-1 \\
i
\end{array}\right) e^{-(x / \beta)^{(n+i+1) \alpha}}\right] .
\end{aligned}
$$

Proof. We know that

$$
\begin{aligned}
g_{m: m} & =m G^{m-1}(x) g(x) \\
& =m \frac{\alpha}{\beta}\left(\frac{x}{\beta}\right)^{\alpha-1} e^{-(x / \beta)^{\alpha}}\left[1-e^{-(x / \beta)^{\alpha}}\right]^{m-1} \text { [Using (29) and (30)] } \\
& =\frac{m \alpha}{\beta}\left(\frac{x}{\beta}\right)^{\alpha-1} \sum_{j=0}^{m-1}(-1)^{j}\left(\begin{array}{c}
m-1 \\
j
\end{array}\right) e^{-(x / \beta)^{(j+1) \alpha}} \text { [on binomial expansion] } .
\end{aligned}
$$

Now using this result in (48) and on algebraic manipulation, we get the required result.

Theorem 6.4. For $n=2,3, \ldots$, the $k^{\text {th }}$ order moment of largest OS for the $p d f(32)$ of quadratic rank TWD is given by

$$
\begin{aligned}
\left(\mu_{n: n}^{(k)}\right)_{T W D 2}= & n(4 / 3)^{n} \beta^{k}\left[\sum _ { i = o } ^ { n - 1 } \sum _ { j = 0 } ^ { n + i - 1 } \frac { ( - 1 ) ^ { i + j } } { ( j + 1 ) 4 ^ { i } } ( \begin{array} { c } 
{ n - 1 } \\
{ i }
\end{array} ) \left\{\left(\begin{array}{c}
n+i-1 \\
j
\end{array}\right)\right.\right. \\
& \left.\left.-\frac{1}{2}\left(\begin{array}{c}
n+i \\
j
\end{array}\right)\right\} \Gamma\left\{\frac{k+\alpha}{(j+1) \alpha}\right\}+\sum_{i=0}^{n-1} \frac{(-1)^{n+i+1}\left(\begin{array}{c}
n-1 \\
i
\end{array}\right)}{2(n+i+1) 4^{i}} \Gamma\left\{\frac{k+\alpha}{(n+i+1) \alpha}\right\}\right] .
\end{aligned}
$$

Proof. For Weibull distribution defined in (29) and (30), we have

$$
g_{m: m}(x)=m G^{m-1}(x) g(x) .
$$

By expanding above expression binomially, we get

$$
\begin{aligned}
g_{m: m}(x) & =m\left(1-\exp \left\{-\left(\frac{x}{\beta}\right)^{\alpha}\right\}\right)^{m-1}\left(\frac{\alpha}{\beta}\right)\left(\frac{x}{\beta}\right)^{\alpha-1} \exp \left\{-\left(\frac{x}{\beta}\right)^{\alpha}\right\} \\
& =\frac{m \alpha}{\beta^{\alpha}} x^{\alpha-1} \sum_{j=0}^{m-1}(-1)^{j}\left(\begin{array}{c}
m-1 \\
j
\end{array}\right) \exp \left\{-\left(\frac{x}{\beta}\right)^{(j+1) \alpha}\right\} .
\end{aligned}
$$

Now $k$ th order moment of largest OS of Weibull distribution (30) is given by

$$
\begin{aligned}
\mu_{m: m}^{(k)} & =\int_{0}^{\infty} x^{k} g_{m: m}(x) d x \\
& =\frac{m \alpha}{\beta^{\alpha}} \sum_{j=0}^{m-1}(-1)^{j}\left(\begin{array}{c}
m-1 \\
j
\end{array}\right) \int_{0}^{\infty} x^{k+\alpha-1} \exp \left\{-\left(\frac{x}{\beta}\right)^{(j+1) \alpha}\right\} d x \text { on using (52). }
\end{aligned}
$$


Since $\int_{0}^{\infty} x^{n} \exp \left\{-(q x)^{m}\right\} d x=\frac{\Gamma\left(\frac{n+1}{m}\right)}{m q^{n+1}}, m, n>0$.

Therefore,

$$
\begin{aligned}
\mu_{m: m}^{(k)} & =\frac{m \alpha}{\beta^{\alpha}} \sum_{j=0}^{m-1}(-1)^{j}\left(\begin{array}{c}
m-1 \\
j
\end{array}\right) \frac{\beta^{k+\alpha}}{(j+1) \alpha} \Gamma\left\{\frac{k+\alpha}{(j+1) \alpha}\right\}, \\
& =m \beta^{k} \sum_{j=0}^{m-1}(-1)^{j}\left(\begin{array}{c}
m-1 \\
j
\end{array}\right) \frac{1}{j+1} \Gamma\left\{\frac{k+\alpha}{(j+1) \alpha}\right\} .
\end{aligned}
$$

Now using (53) in (49) for $m=n+i$ and $m=n+i+1$ then the $k$ th order moment of largest OS for the $p d f$ (32) of quadratic rank TWD is given by

$$
\begin{aligned}
\left(\mu_{n: n}^{(k)}\right)_{T W D 2}= & n(4 / 3)^{n} \sum_{i=0}^{n-1}(-1 / 4)^{i}\left(\begin{array}{c}
n-1 \\
i
\end{array}\right)\left[\beta^{k} \sum_{j=0}^{n+i-1}(-1)^{j}\left(\begin{array}{c}
n+i-1 \\
j
\end{array}\right) \frac{1}{j+1} \Gamma\left\{\frac{k+\alpha}{(j+1) \alpha}\right\}\right. \\
& \left.-\beta^{k} \frac{1}{2} \sum_{j=0}^{n+i}(-1)^{j}\left(\begin{array}{c}
n+i \\
j
\end{array}\right) \frac{1}{j+1} \Gamma\left\{\frac{k+\alpha}{(j+1) \alpha}\right\}\right] \\
= & n(4 / 3)^{n} \beta^{k} \sum_{i=0}^{n-1}(-1 / 4)^{i}\left(\begin{array}{c}
n-1 \\
i
\end{array}\right)\left[\sum_{j=0}^{n+i-1}(-1)^{j}\left\{\left(\begin{array}{c}
n+i-1 \\
j
\end{array}\right)-\frac{1}{2}\left(\begin{array}{c}
n+i \\
j
\end{array}\right)\right\} \frac{1}{j+1} \Gamma\left\{\frac{k+\alpha}{(j+1) \alpha}\right\}\right. \\
& \left.+\frac{(-1)^{n+i+1}}{2(n+i+1)} \Gamma\left\{\frac{k+\alpha}{(n+i+1) \alpha}\right\}\right] .
\end{aligned}
$$

Now on algebraic manipulation, we have

$$
\begin{aligned}
= & n(4 / 3)^{n} \beta^{k}\left[\sum_{i=0}^{n-1} \sum_{j=0}^{n+i-1} \frac{(-1)^{i+j}}{(j+1) 4^{i}}\left(\begin{array}{c}
n-1 \\
i
\end{array}\right)\left\{\left(\begin{array}{c}
n+i-1 \\
j
\end{array}\right)-\frac{1}{2}\left(\begin{array}{c}
n+i \\
j
\end{array}\right)\right\} \Gamma\left\{\frac{k+\alpha}{(j+1) \alpha}\right\}\right. \\
& \left.+\sum_{i=0}^{n-1} \frac{(-1)^{n+i+1}\left(\begin{array}{c}
n-1 \\
i
\end{array}\right)}{2(n+i+1) 4^{i}} \Gamma\left\{\frac{k+\alpha}{(n+i+1) \alpha}\right\}\right] .
\end{aligned}
$$

Hence the theorem.

Table 2 Distributional characteristics of largest OS of TWD2 (48) for different parametric values and sample size.

\begin{tabular}{cccccccccccccc}
\hline \multirow{n}{*}{} & \multicolumn{3}{c}{$\boldsymbol{\alpha}=\mathbf{1 . 5} \boldsymbol{\beta}=\mathbf{0 . 5}$} & \multicolumn{3}{c}{$\boldsymbol{\alpha}=\mathbf{0 . 5}, \boldsymbol{\beta}=\mathbf{0 . 9}$} & \multicolumn{2}{c}{$\boldsymbol{\alpha} \mathbf{2 . 0 , \beta}=\mathbf{2 . 0}$} \\
\cline { 2 - 13 } & Mean & Var & Skew & Kurto & Mean & Var & Skew & Kurto & Mean & Var & Skew & Kurto \\
\hline $\mathbf{2}$ & 0.2533 & 0.3362 & 1.2027 & 1.8339 & 2.0547 & 23.3801 & 25.5977 & 56.0054 & 0.8056 & 3.9894 & 1.4560 & 1.8314 \\
$\mathbf{3}$ & 0.3605 & 0.4288 & 0.3614 & 1.0651 & 2.8803 & 32.3545 & 18.0286 & 41.2666 & 1.1767 & 5.1747 & 0.4353 & 0.9362 \\
$\mathbf{4}$ & 0.4428 & 0.4923 & 0.0961 & 0.8081 & 3.6262 & 40.2543 & 14.0814 & 33.6843 & 1.4542 & 5.9506 & 0.1244 & 0.6501 \\
$\mathbf{5}$ & 0.5112 & 0.5401 & 0.0097 & 0.7112 & 4.3217 & 47.2606 & 11.6460 & 29.0873 & 1.6804 & 6.5160 & 0.0184 & 0.5415 \\
$\mathbf{6}$ & 0.5708 & 0.5779 & 0.0053 & 0.6877 & 4.9808 & 53.4868 & 9.9987 & 26.0429 & 1.8741 & 6.9525 & 0.0013 & 0.5097 \\
$\mathbf{7}$ & 0.6242 & 0.6084 & 0.0482 & 0.7061 & 5.6114 & 59.0147 & 8.8196 & 23.9196 & 2.0454 & 7.3006 & 0.0325 & 0.5195 \\
$\mathbf{8}$ & 0.6731 & 0.6334 & 0.1233 & 0.7526 & 6.2186 & 63.9072 & 7.9448 & 22.3947 & 2.2003 & 7.5835 & 0.0946 & 0.5553 \\
$\mathbf{9}$ & 0.7184 & 0.6539 & 0.2231 & 0.8201 & 6.8060 & 68.2150 & 7.2812 & 21.2858 & 2.3425 & 7.8156 & 0.1789 & 0.6095 \\
$\mathbf{1 0}$ & 0.7608 & 0.6706 & 0.3435 & 0.9048 & 7.3763 & 71.9799 & 6.7727 & 20.4830 & 2.4746 & 8.0067 & 0.2807 & 0.6779 \\
\hline
\end{tabular}




\section{CONCLUSIONS}

In this paper we have generated new generalized transmuted family of distributions (TDn). Some generalized transmuted distributions available in literature are found as particular cases of our transmuted family of distributions. These new generalized transmuted families of distributions are applied to Weibull distribution to find generalized rank map transmuted Weibull distribution (TWDn). Simulated hazard function, pdf curves, and some distributional characteristics such as mean, variance, skewness, and kurtosis for some specific parametric values of generalized transmuted families of Weibull distribution are presented in Figures 1-8 and in Table 1 respectively to make a comparative study among changes of rank maps. Also simulated quadratic ranked transmuted largest os's distributional characteristics are studied and presented in Table 2. These new distributions are more flexible and skewed compare to ordinary Weibull distribution. Flexibility prominently increases as degree of rank of transmutation map increases. These are observed in pdf curves (Figure 4-8) plotting as well as in distributional characteristics presented in Table 1. It is observed that the transmuted distributions are more flexible to model real data, since the data can present a high degree of skewness and kurtosis. If someone is interested to locate more flexible and higher degree of skewed distribution can explore this generalized transmuted family of distributions for future use.

\section{CONFLICT OF INTEREST}

The authors declare that there are no conflicts of interest regarding the publication of this paper.

\section{AUTHORS' CONTRIBUTIONS}

All authors have read and agreed to the published version of the manuscript.

\section{ACKNOWLEDGMENTS}

The author would like to thank the Editor-in-Chief, and the anonymous referees for their careful reading and constructive comments and suggestions which greatly improved the presentation of the paper.

\section{REFERENCES}

1. C. Lee, F. Famoye, A. Alzaatreh, Wiley Interdiscip. Rev. Comput. Stat. 5 (2013), 219-238.

2. K. Pearson, Philos. Trans. R. Soc. Lond. A. 186 (1895), 343-414.

3. N.L. Johnson, Biometrika. 36 (1949), 149-176.

4. J.W. Tukey, The Practical Relationship Between the Common Transformations of Percentages of Counts and of Amounts, Technical Report 36, Statistical Techniques Research Group, Princeton University, Princeton, NJ, USA, 1960.

5. N.L. Johnson, S. Kotz, N. Balakrishnan, Continuous Univariate Distributions, vol. I, second ed., John Wiley \& Sons, Inc., New York, NY, USA, 1994. https://1lib.in/book/1121948/3b61a8

6. N. Eugene, C. Lee, F. Famoye, Commun. Stat. Theory Methods. 31 (2002), 497-512.

7. M.C. Jones, Stat. Methodol. 6 (2009), 70-81.

8. A. Alzaatreh, C. Lee, F. Famoye, Metron. Metron. 71 (2013), 63-79.

9. M. Bourguignon, I. Ghosh, G.M. Cordeiro, J. Probab. Stat. (2016), 7208425.

10. A.Z. Afify, G.M. Cordeiro, H.M. Yousof, A. Alzaatreh, Z.M. Nofal, J. Data Sci. J. Data Sci. 14 (2016), 245-270.

11. D.C.T. Granzotto, F. Louzada, N. Balakrisnan, J. Stat. Comput. Simul. 87 (2017), 2760-2778.

12. K.A. Al-Kadim, M.H. Mohammed, J. Univ. Babylon Pure Appl. Sci. 25(3) (2017), 862-876. http://www.purescience.uobabylon.edu.iq/fileshare/ articles/repository1_publication1493_18_513.pdf

13. K. Jayakumar, M.G. Babu, Statistica. 77 (2017), 251-276.

14. A. Mahdavi, D. Kundu, Commun. Stat. Theory Methods. 46 (2017), 6543-7557.

15. M. Alizadeh, F. Merovci, G. Hamedani, Hacet. J. Math. Stat. 46 (2017), 645-667.

16. K.A. AL-Kadim, J. Univ. Babylon Pure Appl. Sci. 26 (2018), 66-74. https://www.journalofbabylon.com/index.php/JUBPAS/article/view/686/497

17. I. Pobočíková, Z. Sedliačková, M. Michalková, MATEC Web Conf. 157 (2018), 1-11.

18. M. Elgarhy, I. Elbatal, M.A. Haq, A.S. Hassan, Ann. Data. Sci. 5 (2018), 565-581.

19. A.Z. Afify, G.M. Cordeiro, H.M. Yousof, A. Saboor, E.M.M. Ortega, E.M.M Hacet, J. Math. Stat. 47 (2018), 365-381.

20. W.T. Shaw, I.R.C. Buckley, in: IMA First Conference on Computational Finance, 2007. arXiv:0901.0434

21. M. Zubair, M.H. Tahir, M.G. Cordeiro, A. Alzaatreh, E.M.M. Ortega, J. Stat Distrib. Appl. 5(1) (2018), 1-17.

22. W. Weibull, Ing. Vetenskapa Acad. Handlinger. 151 (1939), 1-45.

23. M. Xie, Y. Tang, Y. Goh, Reliab. Eng. Syst. Saf. 76 (2002), 279-285.

24. M. Bebbington, C.D. Lai, R. Zitikis, Reliab. Eng. Syst. Saf. 92 (2007), 719-726.

25. M. Nassar, A. Alzaatreh, O. Abo-Kasem, M. Mead, M. Mansoor, Ann. Data. Sci. 5 (2018), 421-436. 
26. A. Jeffrey, H.H. Dai, Handbook of Mathematical Formulas and Integrals, forth ed., Academic Press, Elsevier, MA, USA, 2008. https://doc.lagout.org/Others/Maths/Calculus/Academic.Press.Handbook.Of.Mathematical.Formula.And.Integrals.4th.Edition.Jan.2008.eBookELOHiM.pdf

27. A.E. Sarhan, B.G. Greenberg, Contributions to Order Statistics, John Wiley \& Sons, New York, NY, USA, 1962.

28. B.C. Arnold, N. Balakrishnan, Relations, Bounds and Approximations for Order Statistics, Lecture Notes in Statistics, vol. 53, Springer-Verlag, New York, NY, USA, 1989.

29. N. Balakrishnan, A.C. Cohen, Order Statistics and Inference: Estimation Methods, Academic Press, San Diego, CA, USA, 1991. https://1lib.in/book/2367374/e2b0e5

30. B.C. Arnold, N. Balakrishnan, H.N. Nagaraja, A First Course in Order Statistics, John Wiley \& Sons, New York, NY, USA, 1992.

31. M.A. Ali, Moments of Order Statistics and Related Results, PhD Thesis, Department of Statistics \& Operations Research, Aligarh Muslim University, Aligarh, India, 1994. https://shodhganga.inflibnet.ac.in/handle/10603/57569

32. H.A. David, H.N. Nagaraja, Order Statistics, John Wiley \& Sons, New York, NY, USA, 2003. 